Journal de la Société des Océanistes

\section{Journal de la Société des Océanistes}

$147 \mid 2018$

La Kanaky Nouvelle-Calédonie a rendez-vous avec I'histoire

\title{
2018. La Kanaky Nouvelle-Calédonie a rendez-vous avec l'histoire... Présentation raisonnée du dossier
}

2018. New Caledonia has a date with History. Reasoned presentation of the file

Isabelle Leblic et Umberto Cugola

\section{CpenEdition}

Journals

Édition électronique

URL : https://journals.openedition.org/jso/9825

DOI : $10.4000 /$ jso.9825

ISSN : $1760-7256$

Éditeur

Société des océanistes

Édition imprimée

Date de publication : 15 décembre 2018

Pagination : 291-308

ISBN : 978-2-85430-136-6

ISSN : 0300-953x

Référence électronique

Isabelle Leblic et Umberto Cugola, « 2018. La Kanaky Nouvelle-Calédonie a rendez-vous avec

I'histoire... Présentation raisonnée du dossier », Journal de la Société des Océanistes [En ligne], 147 |

2018, mis en ligne le 15 décembre 2018, consulté le 27 septembre 2021. URL : http://

journals.openedition.org/jso/9825; DOI : https://doi.org/10.4000/jso.9825

Journal de la société des océanistes est mis à disposition selon les termes de la Licence Creative Commons Attribution - Pas d'Utilisation Commerciale - Pas de Modification 4.0 International. 


\section{La Kanaky Nouvelle-Calédonie a rendez-vous avec l'histoire... Présentation raisonnée du dossier}

par

Isabelle Leblic ${ }^{*}$ avec Umberto Cugola*

Le Journal de la Société des Océanistes revient une nouvelle fois sur la Nouvelle-Calédonie que nombre d'entre nous appellent désormais Kanaky Nouvelle-Calédonie. Plusieurs volumes lui ont été consacrés depuis les débuts du $J S O^{1}$ et, à chaque fois, pour marquer une étape de l'histoire calédonienne.

2018 est une date importante dans l'histoire de ce pays puisque c'est celle du rendez-vous avec l'horizon vers lequel la société, les forces politiques locales et l'État français s'étaient projetés trente ans (accords de Matignon-Oudinot) puis vingt ans (accord de Nouméa) plus tôt. C'est l'année d'un référendum ${ }^{2}$ qui débute le processus de sortie de l'ère de l'accord de Nouméa (1998). Elle va engager ses habitants, ses citoyens puisque ce sont eux qui vont voter, vers des possibles dont les enjeux sont déterminants en termes de choix de modèle de société et de sa viabilité. Le Journal de la Société des Océanistes ne pouvait donc pas manquer ce rendez-vous. La force et l'intensité de ces enjeux convoquant la réflexion, les contribu- teurs de ce dossier ont été invités à penser les perspectives selon lesquelles l'avenir de la NouvelleCalédonie pourrait se dessiner. Ces perspectives ont été envisagées depuis un bilan que chacun pourra établir à différents niveaux, en fonction des centres d'intérêts qui ont motivé sa contribution et des divers champs disciplinaires qui inspirent son argumentaire : quel chemin parcouru, quelles avancées produites, quels déséquilibres corrigés, partiellement rattrapés ou encore défaillants? Nous les voulions dans les divers champs du monde social que nous avions proposés, mais tous ne sont malheureusement pas couverts ${ }^{3}$.

Ainsi, parmi les questions que nous avons souhaité voir traitées dans nos colonnes, certaines l'ont été, d'autres non. D'aucuns pourront regretter aussi que tous les points de vue ne soient pas exprimés. Nous n'avons par exemple pas reçu de contributions scientifiques contenant une argumentation solide en faveur du non au référendum et nous le regrettons. Nous profitons de cette présentation raisonnée des différentes contributions

1. Le dernier en date, mais de façon accessoire, est le volume 136-137 : La part d' " immatériel " dans la culture "matérielle " paru à l'occasion de l'exposition Kanak. L'art est parole au musée du quai Branly. Plus tôt, était paru le volume 117 : Nouvelle-Calédonie, 150 ans après la prise de possession, qui était plus directement axé sur la situation du pays après 150 ans de présence française en Nouvelle-Calédonie. Et le tout premier a été le volume 9, paru en 1953 pour le centième anniversaire de l'annexion du pays par la France (Leblic, $2003: 135-145$ ). Enfin, on notera que le volume 100-101 de 1995, date d'un double anniversaire, celui des 50 ans de la $\mathrm{SdO}$ - et donc du centième numéro du $J S O$ - et celui du premier festival des arts mélanésiens de Nouvelle-Calédonie, Melanesia 2000, organisé en 1975, comportait un important dossier sur cet événement qui fut très important pour le pays. Au total, ce sont 228 articles qui sont déjà parus à propos de la Nouvelle-Calédonie dans les colonnes du JSO auxquels il faut ajouter les 90 comptes rendus de lectures d'ouvrages portant sur le pays.

2. Au moment où nous mettons sous presse ce volume, les résultats du référendum du 4 novembre sont connus, Isabelle Leblic y revient donc « En guise de conclusion... " (pp. 577-588).

3. Soit par manque de temps pour certains qui avaient annoncé une contribution qu'ils n'ont pas pu finaliser, soit par manque de réponse de spécialistes de certaines disciplines, en raison sans doute de la multiplication des initiatives dans cette période pré-référendaire et de l'existence de plusieurs autres publications.

* Anthropologue, DR1 au LACiTO CNRs à Villejuif, leblic@vjf.cnrs.fr

** Docteur en géographie du développement, formateur à l’IfRAss à Toulouse, ucugola@gmail.com 


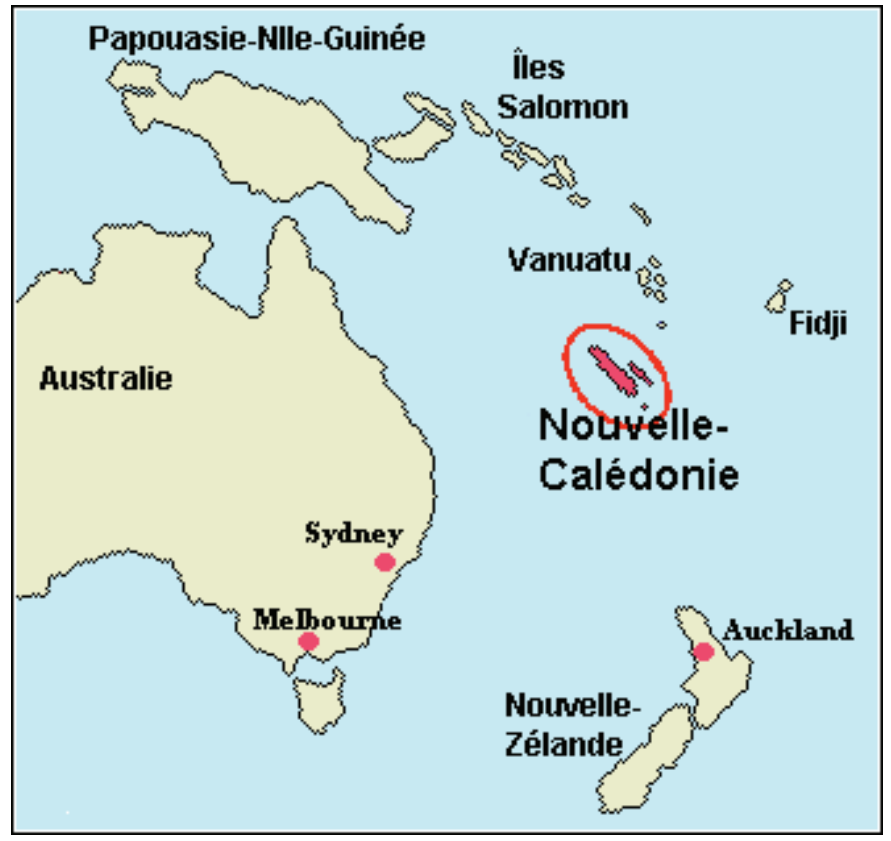

CARTe 1. - La Nouvelle-Calédonie dans son environnement régional (http://www.axl.cefan.ulaval.ca/pacifique/ncallgeo.htm)

pour remercier tous les lecteurs qui ont commenté les textes et ont permis de les améliorer, et dont les remarques ont aussi contribué à étoffer cette présentation.

\section{Domaine politique et institutionnel}

Sur le plan politique et institutionnel, comment penser les concepts clés permettant de saisir un fil signifiant dans l'évolution historique en cours ? La décolonisation, par exemple, a-t-elle aujourd'hui le même sens qu elle avait après-guerre ? Et donc, l'indépendance est-elle toujours d'actualité ? Y at-il actuellement un ou plusieurs nationalismes calédoniens et quel sens lui/leur donner au regard de l'histoire de ce concept, de son actualité sur le continent européen, de la traduction qu'il subit dans l'environnement océanien? Ces nationalismes visent-ils une pleine souveraineté, un système d'État associé ou un principe d'autonomie très large? Comment, dans tous ces modèles possibles, envisage-t-on de régler le passif colonial et la revendication identitaire kanak ? À un autre niveau, quelle feuille de route institutionnelle, politique, voire constitutionnelle organisera la suite à donner au processus de décolonisation? Plusieurs auteurs ont tenté d'apporter des réponses à ces questions. Sémir Al Wardi, dans sa contribution "Changer la politique : le concept de "pays associé " comme solution ? " (pp. 309318), propose une réflexion sur le concept de pays associé. Hamid Mokaddem, dans son article
«L'accord de Nouméa. Pratique de discours et forclusion de la souveraineté de Kanaky" (pp.319-328), pense que le discours contenu dans l'accord de Nouméa tout comme ceux qu'il suscite amènent à la forclusion de la souveraineté et de la notion même de Kanaky et de peuple kanak. Isabelle Leblic (pp. 329-350) s'interroge sur le " rééquilibrage " et la décolonisation en revenant sur 40 ans de va-et-vient institutionnels et sur colonialisme versus souveraineté en Nouvelle-Calédonie. Enfin, Caroline Graille, dans son article "Passions identitaires et communautés de destin : une utopie calédonienne? " (pp. 351-364), approche la communauté de destin en lien avec les passions identitaires et se demande s'il faut voir ce concept comme une utopie calédonienne. La retranscription et traduction par Leah Pappas, étudiante en linguistique à l'université de Mānoa, de la table ronde qui a eu lieu à Hawai' i-Mānoa en février 2017, "Destins (pas) communs " (pp. 563-574), présentée en Miscellanées, apporte aussi des éclairages sur ces questions, un an et demi avant la tenue du référendum.

\section{Domaine économique}

Sur le plan économique, nombreux sont ceux qui doutent encore de la viabilité et de la faisabilité d'un projet d'indépendance ou de large autonomie. Les limites sont-elles uniquement matérielles, de l'ordre des ressources économiques, des compétences humaines et, plus globalement, d'un niveau de technicité encore trop bas ? Dans quelle mesure prendre en compte, sinon comme limite, du moins comme enjeu, la question de la pratique et de la culture démocratique? Une part des enjeux se situe ici dans la question de la monnaie : $\mathrm{FCFP}^{4}$, monnaie locale, euro ? Laïsa Roi reprend ici le dossier de la monnaie ("Quelle monnaie pour la Kanaky Nouvelle-Calédonie après 2018 ? ", pp. 427-440). Que deviendraient les transferts de l'État? Comment se renégocientils ? Quels choix économiques, stratégiques aussi, au regard des ressources disponibles et du poids de l'industrie du nickel ? Franck Enjuanes aborde la question minière sous l'angle de l'évolution de la fiscalité minière calédonienne ("Évolution des principes et des rendements de la fiscalité minière calédonienne, une perspective historique ", pp. 413-426). Quelle place pour l'écologie, le tourisme, l'agriculture? L'autosuffisance

4. Au départ "franc des colonies françaises du Pacifique " (décembre 1945), devenu franc de la "communauté financière du Pacifique ", puis « change franc Pacifique ». Aujourd'hui, 1000 FCFP = 8,38 €. 
alimentaire est-elle une utopie? Quels ancrages dans le système économique régional et, au-delà, français, européen et mondial ? Enfin, on s'interrogera aussi sur le poids de l'économie dans son rapport au projet de société, sur sa contribution dans le rattrapage ou non des déséquilibres entre territoires, groupes sociaux et culturels. Le projet ambitieux d'une décolonisation réussie est-il soutenu par un non moins ambitieux modèle économique et social néo-calédonien ? Parmi ceux présents aujourd'hui, Mokaddem, Roi, Rallu, Leblic et Enjuanes traitent chacun à leur façon de ces questions.

En matière économique, outre l'instauration d'une véritable politique fiscale plus juste et redistributive, il demeure une question d'importance pour l'avenir du pays, l'indexation des salaires, qui " reste au cour de la colonie de peuplement" (Anonyme, $2018:$ 7). Vestige de la France coloniale, les indices de majoration des salaires des fonctionnaires ont un impact sur le pays qui a de forts effets négatifs : " hausse des inégalités, niveau trop élevé des prix et de la dépense publique, surévaluation de la monnaie, choix d'investissement dispendieux dus à l'argent "facile" " (ibid.). L'auteur anonyme de l'article "Désindexation, quel impact pour notre pays ? " dans ce récent article de la revue La voix de Kanaky indique que " La France donne beaucoup, mais mal» :

«En quinze ans, les dépenses de l'État ont doublé, même si cette part des transferts dans le PIB baisse tendanciellement " (ibid.)

En résumé, un PIB de 1000 milliards de FCFP (8,38 milliards d'euros); 155,5 milliards de transferts de la France ( $15 \%$ du PIB) dont :

« $63 \%$ de traitements et de pensions et seulement $24 \%$ de dotations et contrats de développement ! [...] D'ailleurs, [...] l'essentiel des transferts, soit 120 milliards de FCPF repart vers la métropole : assurances-vie, cotisations retraites du secteur privé [...], placements immobiliers en France et en Australie [...]. » (ibid.)

\section{Domaines identitaire et culturel}

Sur les plans identitaire et culturel, comment envisager le "vivre ensemble" ? Que produit la société calédonienne: multiculturalisme, pluralisme, ethnicité, créolisation, logiques métisses, syncrétisme? Quels sont les processus d'altérité qui animent la mosaïque ethnique - dont on peut/doit s'interroger sur la manière dont elle est construite -, qui la mettent en tension ou produisent une sublimation des particularismes dans un " être ensemble » ? Plusieurs auteurs apportent ici un éclairage, chacun en son domaine, lesquels, mis bout à bout, permettent de dessiner divers scénarios. Quel rôle attribuer à la statistique et aux études démographiques pour observer et rendre compte de cette diversité ? Jean-Louis
Rallu, dans son article "Retour sur les notions de "rééquilibrage" et "décolonisation" " (pp. 397412), reprend en démographe les données statistiques pour faire le point sur le rééquilibrage et la possible/impossible décolonisation. Quelle est la contribution des politiques identitaires et culturelles dans les dynamiques du lien social ? En quoi ont-elles corrigé les déséquilibres et les effets de domination hérités de la colonisation? Et quel rôle l'école joue-t-elle à ce niveau ? Jusqu'à quel point est-elle partenaire d'une communauté de destin de la mosaïque ethnique ? Existe-t-il un modèle d'école propre à la société calédonienne ?

Benoit Carteron et Umberto Cugola s'attachent à divers aspects de la société kanak en contexte urbain. Benoît Carteron, dans « Habiter un quartier périurbain pluriculturel. Katiramona-Nondoué (Dumbéa) » (pp. 441-456), décrit la cohabitation au sein d'un quartier péri-urbain situé sur la commune de Drumbéa dans le Grand Nouméa. Umberto Cugola, quant à lui, à partir d'un retour au pays en famille en pays djubéa (à La Conception), présente l'espace de la coutume dont l'importance reste actuelle dans cette zone du Grand Nouméa toujours aux prises avec de nombreuses violences ("Espace de la coutume et enjeux civilisationnels. Témoignage sur un retour en pays djubéa ", pp. 457-472). Rachid Oulahal, Zohra Guerraoui et Patrick Denoux appliquent la notion de diaspora, pour les descendants de déportés algériens et kabyles ("Entre mémoire collective et émergence diasporique. Le cas des descendants d'Algériens en Nouvelle-Calédonie ", pp. 373-382). Christophe Sand revient aussi sur les descendants d'Algériens à partir d'une expérience plus personnelle ("Être descendants d'exilés algériens à Caledoun: réflexions sur la place des arabes calédoniens dans l'histoire », pp. 365-372) et Claude Grin sur ceux des Japonais ayant fait souche au pays ( $"$ Le retour des ancêtres japonais ", pp. 383-396). Dominik Bretteville (" "Je ne sais pas ce que c'est, la coutume" : La coutume kanak par-delà tradition et modernité ", pp. 473-486), Matteo Gallo et Anna Paini ("Gérer le passé pour façonner l’avenir à Wëtë et Drueulu ", pp. 487-500) reviennent chacun à sa façon sur la coutume, son passé et son avenir, dans des contextes divers : Lifou pour Anna Paini, la Grande Terre pour D. Bretteville (chez les Paimboa) et Matteo Gallo (chez les Paicî). Eddy Banaré, à partir des "Images et textes d'Ataï (1969-2016). Élaboration de discours politique kanak», pp. 515-528), montre comment la figure d'un "héros" kanak luttant contre la colonisation a servi de symbole pour la revendication identitaire kanak.

Dans son article "Une école calédonienne en quête d'identité. Modèle français, adaptation, contextualisation, émancipation ?" (pp. 501514), Stéphane Minvielle traite de la question des langues et de l'école en lien avec l'identité. Il revient sur le projet éducatif calédonien voté en 


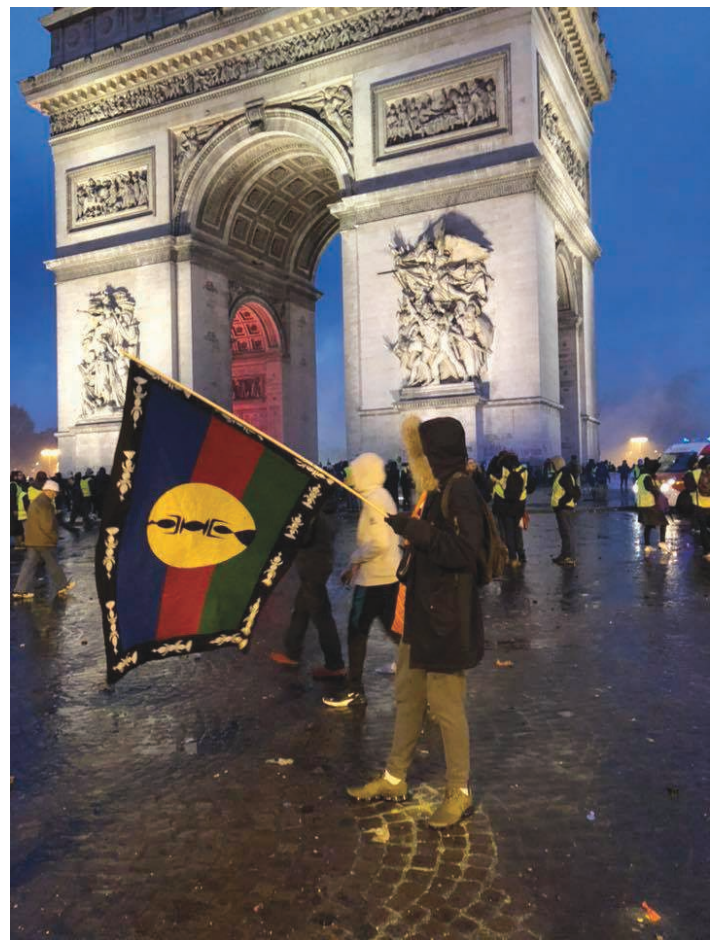

Рното 1. - «Affichons nos couleurs " place de l'Étoile à Paris, 2/12/2018 (Facebook)

2016 par le congrès. Durant ce «temps long octroyé ", l'école calédonienne a vécu plusieurs mutations. Les revendications identitaires culturelles et linguistiques autochtones se sont rapidement ancrées dans le système scolaire. Si bien que l'enseignement de certaines langues et de la culture kanak est aujourd'hui intégré dans le curriculum de formation des enseignants et des programmes scolaires 5 .

\section{Domaine des politiques sociales}

Sur le plan général des politiques sociales liées à la famille, l'enfance, la jeunesse et les personnes vulnérables, la structuration, ces dernières décennies, d'un champ professionnel de travailleurs sociaux est venue se substituer aux œuvres laïques ou religieuses, voire aussi aux solidarités communautaires des tribus et des clans. Comment, dans un projet de société calédonien, inclure et penser la famille, espace par excellence de la transmission des valeurs et des normes qui fondent un vivre ensemble? Comment penser aussi la vulnérabilité des personnes, comme la jeunesse en difficulté ? Comment mettre en place des politiques sociales sans transposer littéralement ce qui vient de la métropole ? Comment alors les inventer et les faire émerger localement? Quelle intervention/accompagnement éducatif et social proposer pour intégrer les occupants de la marge, disqualifiés par le cour dominant du système? À part Umberto Cugola, qui évoque rapidement le sujet dans son article (voir aussi Cugola, 2017) tout comme Benoît Carteron qui parle de "sociabilité ordinaire [...] familiale [...] et de voisinage ", nous n'avons pas ici d'articles traitant ces questions qui sont pourtant d'importance pour l'avenir du pays. Le problème récurrent de la violence des jeunes est un des éléments sur lesquels jouent les anti-indépendantistes pour prédire, sans réel élément d'analyse, un chaos en cas d'indépendance, généralisant les situations de Saint-Louis et La Conception à tous les jeunes, kanak et périurbains en particulier.

Une réflexion est également à mener sur la coutume et les structures coutumières: les tribus, les clans, les chefferies, les terres coutumières, les groupements de droit particulier local (GDPL), le sénat coutumier. Dans une société calédonienne qui connait un dualisme juridique avec le droit commun et un droit particulier, ce sont bien deux systèmes institutionnels qui sont à penser en articulation l'un à l'autre. Or cette nécessité ne s'impose pas uniquement en raison d'un besoin de reconnaissance de la part des Kanak; elle est impérative car elle soutient les grands équilibres sociaux et territoriaux du pays dans son ensemble, comme le montre ici Cugola pour les tribus de Saint-Louis et de La Conception. Quelle légitimité, quelle reconnaissance, quel statut pour la chefferie ? À quelle échelle de territoire faut-il envisager une ou plusieurs autorités coutumières? Quelles sont les attributions d'une autorité coutumière à différents niveaux : clan, tribu, district, sénat...? Comment ces autorités coutumières s'articulentelles entre elles et au pouvoir politique? Quelles rôles doivent-elles prendre et assumer vis-à-vis de la chose publique, du bien commun, de la vie de la cité ? Et, à partir de là, sommes-nous sur la voie d'un modèle démocratique singulier capable de produire un consensus entre le particulier et l'universel ? Dans son article, Dominik Bretteville traite de la question à un niveau théorique large. Enfin, un dernier questionnement renvoie à la coutume elle-même. Si celle-ci est souvent menacée d'instrumentalisation, elle est bien autre chose que cela puisqu'elle s'adapte aux hommes et non l'inverse. La profondeur de son message cultive une universalité vécue, elle inclut sans cesse l'ailleurs et le différent. Â travers les discours de la coutume, à travers la pensée de certaines figures

5. Certains textes reçus n'ont pu être finalisés pour ce volume et paraîtront donc fin 2019, dans un mini dossier suivant celui-ci où deux autres articles reprendront la question de l'école : Elatiana Razafi présentera un panorama des langues de Nouvelle-Calédonie et la façon dont les jeunes générations, celles nées depuis les accords, les perçoivent ; Eddy Wadrawane nous exposera comment les deux accords politiques (Matignon-Oudinot de 1988 et Nouméa de 1998), ont modifié le paysage calédonien. De même, Pierre-Christophe Pantz se demandera s'il est approprié de parler de diaspora dans le cadre des migrations internes kanak en Nouvelle-Calédonie. 
kanak connues ou reconnues, peut-on postuler l'existence d'une philosophie en lien avec la coutume? Quel message contient-elle et qu'a-t-elle à dire à des hommes préoccupés par une communauté de destin? Sur ce thème, nombre d'articles (notamment ceux de Graille, Carteron, Leblic, Cugola et Bretteville) apportent d'intéressantes pistes de réflexion.

\section{Présentation raisonnée des textes}

Dans son texte, S. Al Wardi rappelle que l'État français a pris pour habitude de brandir la constitution comme une limite à ne pas dépasser. Dans la mesure où la constitution reflète l'histoire politique d'un État ainsi que sa culture politique et juridique, les peuples vivant outre-mer ne se sentent pas toujours liés à cette vision du politique. Dès lors, le concept de "pays associé ", dont l'auteur dit qu'il est inconnu dans le droit français, est fréquemment désigné comme l'une des solutions institutionnelles pour la NouvelleCalédonie. Dans la société calédonienne éclatée, le "vouloir-vivre ensemble " suppose un dialogue des hommes entre eux sur le territoire, porté par la tolérance, sans excès de ressentiment, une sorte de bricolage politique, autre mot donné à l'audace. Est-ce alors une solution?

Ce texte nous invite à nous projeter dans les modèles institutionnels possibles pour la Nouvelle-Calédonie et s'appuie donc pour l'essentiel sur les sciences juridiques et politiques. C'est principalement l'analyse comparative qui fonde l'argumentaire en faveur d'une solution politique pour l'après-référendum de 2018. Une comparaison nous est proposée de modèles de rapports entre un État et ses dépendances : en France, le principe des collectivités/assimilation et au Royaume-Uni, celui du "self-government". Comparatisme élargi à des " pays » insulaires de la région : Nouvelle-Calédonie, Polynésie française, îles Cook, îles sous tutelle des Etats-Unis... Ce contexte comparatiste met en débat plusieurs options envisagées par l'auteur (État associé, départementalisation, collectivité, pays associé) tout en mettant aussi en question les capacités de la République à s'extraire de son cadre constitutionnel trop étroit pour inclure la réalité historique et culturelle des outremers.

Pour certains (A. Mawyer, comm. pers., juillet 2018), dire que "le concept de "pays associé" [est] inconnu du droit français..." est une position difficile à tenir si l'on prend en compte, par exemple, le long cadre historique et politique des relations entre la France et Andorre ou entre la France et Monaco. En effet, l'onu utilise Andorre et Monaco comme des exemples classiques d'États associés, qui ont servi de précédent pour les États associés du Pacifique devenus tels avec leurs puissances colonisatrices que sont la Nou-
velle-Zélande, l'Australie et les États-Unis. À Andorre, le président de la République française est à la tête de l'État suite au contrat de droit féodal, le paréage de 1278 et 1288 , qui concédait le trône d'Andorre aux deux co-suzerains qu'étaient l'évêque catalan d'Urgell et le comte de Foix ; les droits du comte de Foix passèrent au cours des siècles à la couronne de France (Henri de Navarre) et, enfin, au président de la République. La principauté d'Andorre est membre de l'onu depuis 1993, mais pas de l'union européenne. La principauté de Monaco, quant à elle, est une monarchie constitutionnelle, placée sous la protection de la France depuis 1860. Et, selon l'article $436 \mathrm{du}$ traité de Versailles renégocié en 2002 et ratifié en 2005, l'existence politique et légale d'un État associé n'est plus impossible dans la Constitution française. Aussi, pourquoi ce qui a été rendu possible pour des États européens ne le serait-il pas pour des territoires français du Pacifique ? À cela, Sémir Al Wardi répond qu'Andorre et Monaco n'ont jamais été des colonies françaises...

Cela nous renvoie au dernier ouvrage du haut fonctionnaire de l'État, ancien magistrat à la Cour des comptes et bon connaisseur de la NouvelleCalédonie, Jean-Luc Mathieu, La Nouvelle-Calédonie sera-t-elle indépendante? (voir compte rendu dans ce volume, pp. 596-599), qui précise que :

«Nous allons analyser ici une politique parée de tous les atouts de la générosité [...] pour inciter une majorité de Néo-Calédoniens à se détourner de toute perspective indépendantiste et opter pour une large autonomie (affectant le moins possible la richesse des plus aisés). Les pages qui suivent entendent expliquer pour quelles raisons et par quels cheminements le référendum, prévu de longue date, en Nouvelle-Calédonie, pour l'automne 2018, ne peut aboutir qu'au rejet de la "pleine souveraineté", c'est-à-dire de l'indépendance, parce que tout a été fait, depuis 1978, pour aboutir à ce résultat. » (2018:9-10)

Après avoir exploré le champ des modèles de société possibles, on comprend que l'option de "pays associé » soit une solution suggérée de façon bien argumentée et loin de tout prosélytisme par S. Al Wardi. On peut cependant se questionner sur cette position consistant à restreindre la revendication kanak à un besoin de reconnaissance identitaire se traduisant par une demande de compensation en tous genres (p. 311). JeanLuc Mathieu (2018; voir aussi CR pp. 596 sq), quant à lui, rappelle à juste titre que la solution pourrait être celle, avancée aussi dans les projets de société de l'uni et de l'UC-FLnKs, d'un Etat indépendant associé :

" cet État, indépendant, en association avec la France, aurait façonné son histoire, qui l'eut peutêtre menée loin de l'idéal de l'Union calédonienne "Deux couleurs, un seul peuple". Peut-être eût-il réussi, malgré les profondes déstabilisations opérées par les colonisateurs puis, ultérieurement, 


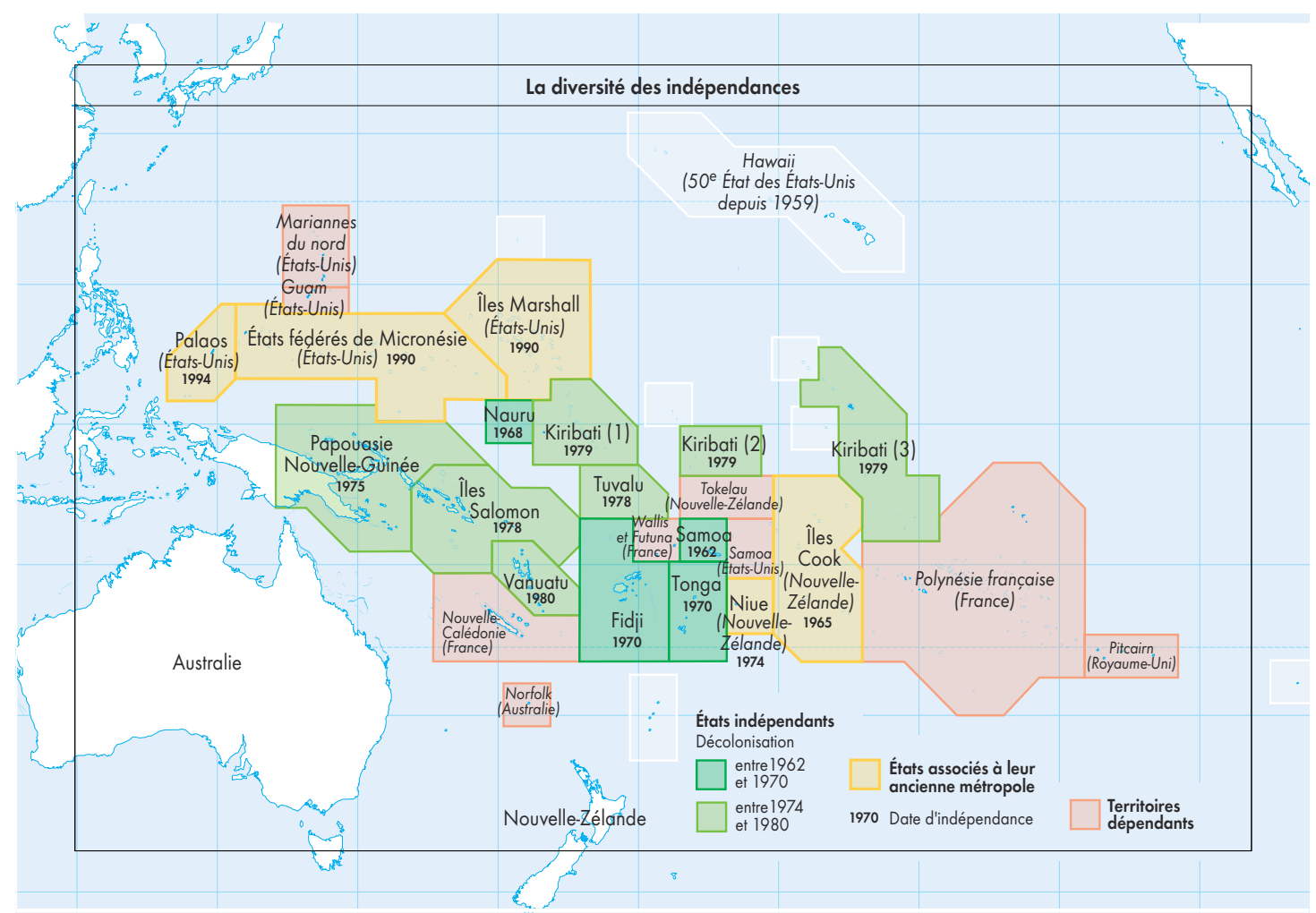

CARTE 2. - Océanie, une décolonsation tardive avec des indépendances récemment acquises (@ cartographie de Cécile Marin in Argounès, Mohamed-Gaillard et Vacher, 2011 : 30).

par l'offensive capitaliste, à construire une société équilibrée, plus égalitaire, vivant selon ses schémas propres, sans courir après un produit intérieur brut moyen toujours plus élevé (la "croissance"), conservant quelques spécificités malgré la tornade de la mondialisation. [...] Mais cet Etat indépendant, associé à une France qui l'aurait honnêtement aidée à construire son propre destin, aurait lui-même façonné son histoire. Encore eût-il fallu qu'il rencontrât d'authentiques décolonisateurs, capables de vouloir qu'un pays essaye de se choisir un autre destin que celui qu'on lui impose et de l'y aider» (Mathieu, 2018 : 121-122).

Que l'autochtonie soit l'un des derniers avatars du colonialisme, comme le souligne Al Wardi, l'analyse est partagée par plus d'un observateur. Mais les Kanak peuvent se saisir de l'autochtonie, de la religion, du politique, des sciences ou de tout autre « objet » si cela les met en relation avec un élément susceptible de concourir à leur émancipation. C'est ce que font d'une certaine façon les militants du Parti travailliste (PT) et de l'USTKE quand ils appellent à la non-participation au référendum au prétexte que celui-ci ne respecte pas strictement les règles de l'onu selon lesquelles seul le peuple autochtone peut se prononcer dans le cadre d'une consultation sur l'accès à l'indépendance. Le motif de ce mot d'ordre est que :

« rien n'a changé, c'est même encore pire [...] la question de fond, la seule question, pour un nationaliste, pour nous indépendantistes, c'est quoi "vouloir l'indépendance" ? c'est vouloir être responsable, c'est vouloir diriger notre pays, [...] c'est un droit, c'est un droit qui est consacré par la charte des Nations unies, c'est un droit qui est consacré par le droit international auquel a souscrit la France, et donc ce droit international est bien spécifié, [...] que le droit des peuples à disposer d'eux-mêmes, c'est uniquement réservé aux peuples colonisés, caractérisés de colonisés. Moi, j'irai voter le jour où ce sera le vrai référendum d'autodétermination, c'est-à-dire que seul le peuple kanak vote [...]. C'est la première fois qu'un peuple colonisé fait une telle concession. Y'a aucun précédent dans l'histoire coloniale, qu'elle soit française ou anglaise [...] on leur reconnaît le statut de victimes de l'histoire. Mais en contrepartie, qu'est-ce qu'on a reçu ? [...] Pour un indépendantiste, c'est quoi la ligne jaune [sic] à ne pas franchir ? C’est quoi le plus important? Le plus important, et tout découle de là, c'est de savoir si l'indépendance est négociable ou pas ; l'indépendance, ce n'est pas négociable [...] » (Extrait de l'intervention de Hnaläne Uregei, $x^{\mathrm{e}}{ }^{\mathrm{e}}$ congrès de l'ustKe, 7-8-9 septembre 2018, Ko we Kara, Nouméa) ${ }^{6}$

6. Après une heure de débat suite aux diverses interventions, les 250 délégués présents passent au vote mais seuls 158 expriment leur choix, soit 37,6\% des délégués : 130 sont pour une non-participation au scrutin du 4 novembre, 16 y sont favorables et 12 choisissent l'abstention (http://ustke.org/actualites/actualite-syndicale/USTKE-:-Le-XVIemeCongres-se-prononce-en-faveur-de-la-non-participation-au-referendum-at_996.html). 
Or, l'autochtonie restera d'autant plus associée au mot "indépendance " que la situation d'inégalité et de domination que subissent les Kanak perdurera: toute autre option institutionnelle (État ou pays associé par exemple) ne relèverait que de l'ordre du discours et serait donc éloignée des pratiques sociales et culturelles.

L'interrogation proposée ci-dessus par Uregei appelle un second niveau de débat. En effet, on a le sentiment que l'option "indépendance" est " éliminée » aujourd'hui. Soit... mais est-ce réellement le cas ? Le rapport des Kanak à cette catégorie étant, lui, bien réel, il doit faire partie des débats d'experts sur l'avenir institutionnel du pays.

On peut avoir le sentiment de retrouver, dans le texte d'Al Wardi, la distinction faite par Castoriadis (dans La Montée de l'insignifiance) à propos du politique comme procédure et de la politique comme fonction instituante (repris dans un texte de J.-B. Paturet, 2001). Dans le politique, nos gouvernants confient à des experts le soin de gérer la vie publique, de les représenter et d'orienter les solutions du "vivre ensemble » en fabriquant des langages techniques et des options émises par des " experts". Le politique aurait tendance à vouloir clôturer le sens et limiter les possibles car il est du côté de la procédure et de la volonté de maîtrise. La politique par contre pourrait se définir :

" comme [une] activité explicite et lucide concernant l'instauration des institutions souhaitables et souhaitées par les citoyens... » (Castoriadis, 1996 : 225)

Autrement dit, il s'agit d'un processus dynamique, dans lequel les solutions se construisent en commun, dans une logique participative et dans des espaces symboliques où éthique et politique se nouent à nouveau. C'est peut-être dans des espaces sociaux à la base de la société que s'élabore un projet de constitution (compris à la fois comme mythe fondateur) relié à des pratiques sociales, à la praxis... loin des options préconçues par les experts. Mais en effet, comment faire exister la politique? Peut-être, en tant " qu'expert ", s'agit-il déjà de mettre au devant de la scène son existence et les enjeux qu'elle suppose. Il nous semble qu'avec leur projet de société et de gouvernance pour la Kanaky Nouvelle-Calédonie souveraine et indépendante, les Kanak indépendantistes ont choisi la politique.

Ces thèmes de la souveraineté et de l'indépendance sont également au cœur des articles d'Hamid Mokaddem et d'Isabelle Leblic. Dans un texte mobilisant plusieurs disciplines et combinant le style du pamphlet et celui de la pensée critique française des années 1960, Mokaddem propose un argumentaire dont émergent trois concepts : la forclusion, la dénégation et le refoulement. L'auteur défend l'idée que l'accord de Nouméa est une pratique discursive qui forclôt la souveraineté de Kanaky. Dire que les accords, en particulier celui de Nouméa, sont un avatar moderne de la colonisation est un constat partagé par nombre d'anti-colonialistes français. Pour ce faire, Mokaddem pointe et analyse un bref passage de l'accord de Nouméa (le point 3 du préambule) qui « forclôt » la souveraineté de Kanaky. Cette analyse est au cour de ce texte et en constitue à notre sens l'un des intérêts majeurs. L'auteur poursuit ce qu'il a déjà fait dans un petit livre pédagogique publié en 2011 sur L'accord de Nouméa pour tous dont nous rendons compte dans nos colonnes plus loin (voir p. 595). Le concept de forclusion dans la psychanalyse du sujet est utilisé ici comme forclusion de l'ordre d'un discours d'un accord politique. Or, la forclusion chez le sujet se situe hors du champ du symbolique alors que la rhétorique du faux (la feinte de la feinte), dans ce qu'il qualifie de forclusion de la souveraineté kanak dans l'accord de Nouméa, reste dans le champ du symbolique. Le signifiant « souveraineté kanak » est remplacé non par un vide hors champ symbolique (c'est le cas avec la forclusion) mais par un autre signifiant: "la souveraineté partagée ». Mokaddem prend parti dans son texte et montre que les mots qui ont disparu du discours ont pu endormir la lutte. Le caractère subversif et provocateur de ce texte est intéressant s'il peut mener à la créativité, à l'espoir et à l'ouverture de nouvelles perspectives... Le mot Kanaky a peut-être disparu de l'ordre du discours, mais les symboles de Kanaky (drapeau, couleurs, teeshirt, casquettes, etc.) circulent librement et de façon décomplexée dans les rues, sur le corps des gens et dans les échanges du quotidien. La campagne pour le référendum a montré l'importance de ces symboles pour la mobilisation des jeunes. Les résultats du 4 novembre 2018 attestent qu'on peut réveiller le mot Kanaky sans crisper les positions des uns et des autres puisque le "Oui » n'est pas le seul apanage des Kanak (voir Leblic dans la Conclusion de ce dossier). Les indépendantistes prônent la "souveraineté partagée ", mais une fois seulement l'indépendance et la souveraineté obtenues par le vote, afin de pouvoir discuter sur un pied d'égalité, d'État à Etat, un partenariat entre Kanaky Nouvelle-Calédonie et la France.

Hamid Mokaddem, tout comme Isabelle Leblic dans le texte qui suit, utilise le concept de " recolonisation ». Si certains pensent que parler de recolonisation implique que la décolonisation de la Nouvelle-Calédonie a déjà eu lieu, pour d'autres, comme ces deux auteurs, ce n'est bien entendu pas le cas. Il s'agit en fait de voir comment les accords ont permis d'accentuer la colonisation dans bien des domaines. On peut assimiler l'arrivée massive, bien que non appréciable en termes quantitatifs, de nouveaux migrants à celle de "colons" d'un nouveau genre, avec une inégalité qui souvent se renforce, avec notamment un écart entre les plus bas et les plus hauts salaires qui se creuse de façon importante. On observe toujours trop d'inégalités en matière économique, sociale, scolaire, etc. comme le montre Jean-Louis 
Rallu dans son article. D'autres auteurs ont aussi pointé ce phénomène, qui n'est pas nouveau, en notant comment le pouvoir colonial, l'État et ses acteurs ont souvent tout fait pour diluer le projet d'indépendance, voire même ont lutté contre une possible souveraineté, malgré les discours affichés (voir notamment Barbançon, 2008 ; Leblic, 1993 ; Mathieu, 2018). Cette articulation entre décolonisation et recolonisation est au cœur de la démonstration selon laquelle les deux forces qui évoquent la souveraineté sont la France, puissance coloniale détentrice de la souveraineté nationale, et le FLNKS qui revendique la souveraineté nationale de Kanaky. Et les loyalistes, la force conservatrice au sens propre, qui représentent le troisième partenaire des accords, veulent depuis toujours que l'idée d'indépendance soit "purgée ", " écrasée " ou " dépassée ".

Pour sa part, Isabelle Leblic propose ici un texte qui fait un tour d'horizon des principaux thèmes intervenant dans l'histoire des quarante dernières années de la Kanaky Nouvelle-Calédonie, en particulier dans les domaines du "rééquilibrage " et de la décolonisation. En revisitant en partie son ouvrage de 1993, elle fait le point sur certaines questions qui y étaient abordées pour les actualiser. Elle revient en particulier sur le rôle de l'anthropologue anticolonialiste en situation coloniale. Comme H. Mokaddem, elle montre en quoi l'expression "décoloniser dans la république " peut parfois recouvrir une recolonisation et que la décolonisation ne peut réellement se faire qu'avec la souveraineté et l'indépendance. Pour préciser ces deux notions, elle revient d'abord sur la Constitution française, puis sur le débat calédonien pré-référendaire, notamment sur la façon dont les sondages ont tenté de discréditer toute idée de vote "Oui ». Écrit comme la plupart des textes publiés ici (hors l'introduction et la conclusion) avant le référendum du 4 novembre 2018 , ce texte reprend l'analyse des votes " indépendantistes " dans les élections précédentes et les compare aux sondages. Pour l'auteure, la table ronde de Nainville-les-Roches comportait déjà la trame des accords qui ont suivi. Pour elle, le développement, puis le rééquilibrage - bien que ce dernier soit très important pour l'avenir du pays peuvent être considérés comme le nouvel alibi de la colonisation. En bref, la Nouvelle-Calédonie est toujours en situation coloniale malgré son passage au statut de territoire d'outre-mer depuis 1946. L'auteure examine aussi les mécanismes de recolonisation à l'œuvre dans ce qui est présenté comme un processus de décolonisation en cours. La mise en œuvre des officiers publics coutumier (OPC), par exemple, semble faire écho à ce que les opérations de développement tentaient déjà de produire dans le domaine foncier (Leblic, 1993) au cours du dernier quart du $\mathrm{Xx}^{\mathrm{e}}$ siècle : renforcer le dispositif de contrôle social en terres coutumières. Le texte présenté en conclusion ( Une Kanaky
Nouvelle-Calédonie souveraine en marche?») permet de compléter ces arguments en revenant sur le référendum depuis la campagne électorale indépendantiste jusqu'aux résultats en forme de "défaite victorieuse " pour les indépendantistes. La chronologie (pp. 529-564), reprise de celle publiée en 2003 mais ici considérablement revue et augmentée, constitue une véritable trame de la colonisation qui permet de recontextualiser dans l'histoire calédonienne, voire française, tous les éléments historiques auxquels se réferent régulièrement les uns ou les autres en parlant d'une nécessaire décolonisation en ce $\mathrm{XXI}^{\mathrm{e}}$ siècle.

Dans son article, Caroline Graille revient à sa façon sur la manière dont l'histoire douloureuse de la colonisation et les transformations statutaires de la Nouvelle-Calédonie ont façonné une société qui se pense avant tout comme fondée sur des différences ethniques et culturelles. Les accords de Matignon-Oudinot de 1988, qui témoignent de la volonté d'une politique de rééquilibrage en faveur des Kanak, puis l'accord de Nouméa de 1998, qui décrète l'injonction politique du "destin commun", ont ravivé les débats sur la place dévolue, d'une part, à l'identité autochtone et, d'autre part, aux autres communautés présentes dans l'espace culturel public calédonien. En s'intéressant à la manière dont des modèles identitaires historiquement antagonistes pourraient donner naissance à une forme d'ancrage géographique et d'appartenance culturelle commune à l'ensemble des citoyens de ce pays, l'auteure nous rappelle que l'anthropologie questionne ici la construction des représentations d'une société à la fois inégalitaire et clivée, métissée et multiculturelle. Mais on se demande quelles traditions, quels héros et quels symboles seront à même d'incarner demain le "vivre ensemble" calédonien dans sa diversité.

Ethnologue de formation, Graille s'intéresse à des objets forts, clairement représentatifs de l'actualité des enjeux du pays: les processus identitaires, le multiculturalisme, l'ethnicité, les références symboliques, le rapport entre culture et politique, la citoyenneté, les théories sur le concept de Nation. Les facteurs historiques entravant l'émergence d'un sentiment national partagé en Nouvelle-Calédonie sont présentés et analysés ici. Ce texte invite donc à la réflexion et à la recherche d'une représentation permettant de situer la (proto-)nation calédonienne entre la nation ethnique (Volksgeist) et la nation civique (républicaine). Les Kanak, ou pour le moins leurs leaders, ont très tôt été animés par l'idéologie ethnoculturelle de la nation selon la Pacific Way, mais on peut se demander si celle-ci intègre suffisamment les autres ethnies du pays et si ces dernières se reconnaissent en elle. Par exemple, une grande partie des Caldoches éprouvent un sentiment d'appartenance au territoire calédonien, avec une singularité revendiquée comme étant une 
particularité ineffable, "quasi ethnique" dans la République. Sur le plan de la subjectivité, ce sentiment de commune appartenance serait lié à une identification commune à un même symbole que la «terre » ou « le pays » traduisent le mieux. Au-delà des clivages, chaque ethnie calédonienne se retrouve autour d'une manière passionnée de dire son pays, de l'intérioriser à travers des sentiments et des affects relevant d'une Pacific Way partagée. Pour autant, ce sentiment diffus d'une commune appartenance n'a pas de traduction politique; la citoyenneté, le peuple calédonien ou plutôt le destin commun restent des principes abstraits qui peinent à mobiliser les Calédoniens. L'auteure prend le parti de montrer que la matérialisation de ces principes en réalités concrètes ne peut se faire que par une action appropriée d'institutions reconnues (Centre culturel Tjiabou, musée de Nouvelle-Calédonie). Pour que cette idée soit opérationnelle, il faut donner à l'institution le sens large que lui donne Durkheim car l'institution politique et officielle de la culture ne peut décider seule de ce qui est représentatif de la commune humanité du peuple. Elle doit forcément être dans une négociation avec des institutions plus ou moins informelles dans l'espace de la coutume (voir le texte de Cugola dans ce volume, pp. 457-472), des familles, de la société civile aussi et des associations, pour valoriser cette action continue et constante qu'elles produisent dans des formes instituées ou constituées de pratiques à travers lesquelles les générations opèrent une transmission sur les savoir-être et le vivre ensemble. Citoyenneté ou destin commun n'auront de réalité concrète que si les représentations culturelles construites autour de ces principes émergent d'un vivre ensemble qui, au fond, est déjà là, dans les pratiques du quotidien. À défaut, ils risquent de n'être que des fictions conceptualisées par des professionnels de la culture ou de la politique et déconnectés de la réalité de ceux qu'ils sont sensés représenter.

Nous assistons depuis quelques années en Nouvelle-Calédonie à un regain d'intérêt identitaire de la part des populations vivant dans l'archipel. Le destin commun en construction ne concerne pas seulement Kanak et Caldoches, mais aussi les descendants d'autres communautés qui ont fait souche dans le pays pendant la colonisation. La Nouvelle-Calédonie, terre coloniale, autrefois de bagne ou d'exil, a connu en effet une phase de transition vers le référendum du 4 novembre 2018 durant laquelle le destin commun a essayé de se construire. Mais celui-ci ne doit pas être cantonné aux seuls derniers accords politiques car, depuis les Événements, en réaction à la demande de reconnaissance d'identité des Kanak, sont également nées des réflexions sur ce que c'est que d'être Calédonien (voir les ouvrages de Louis-José Barbançon, Jean-Pierre Devillers, Éric
Douyère, Ismet Kurtovitch... ou le collectif Etre Calédoniens aujourd'hui).

Dans la contribution de Christophe Sand, à propos des "Arabes " calédoniens, c'est principalement d'histoire dont il est question. L'auteur nous fait partager une histoire incarnée et vécue, avec la profondeur d'un témoignage personnel. En tant qu'enfant du pays lui-même, Umberto Cugola, qui a connu quatre années d'internat au Sacré-Cour de Bourail (1983-1986), fief de la communauté arabo-calédonienne, fait part des résonances bouleversantes qui lui sont venues à la lecture de ce texte :

«Des souvenirs de ces amis d'enfance au collège de Bourail, à l'époque des Événements, avec lesquels nous, les Kanak, n'étions pas d'accord. Pour nous, ils étaient tous blancs : les Boufenèche, les Kadour, les Kader, etc. Jamais, avant la lecture de ce texte, je ne m'étais représenté à quel point nous étions semblables. " (comm. pers., mars 2018)

Cette proximité issue d'un imaginaire social et politique commun mais anesthésiée par la domination coloniale, Christophe Sand la met en évidence en nous livrant sa propre histoire.

Ainsi, des points de débat et de réflexion émergent. Par exemple, la position médiatrice (entre Kanak et Caldoches) avancée par Sand de la communauté arabo-calédonienne est-elle si unique ? On peut avoir en tête le rôle qu'a pu jouer Jean-Pierre Aïfa, homme politique se revendiquant de cette communauté et engagé très tôt aux côtés des indépendantistes (voir Barbançon, 2008 et CR dans ce volume, pp. 592-595). Il existe des exemples individuels similaires dans toutes les communautés, par exemple André Dang pour les Vietnamiens, Louis-José Barbançon ou Jacques Violette pour les Caldoches, anciens de la Fédération pour une nouvelle société calédonienne (FNSC) ou du Parti socialiste kanak (PSK), Sako Aloisio du Rassemblement démocratique océanien (RDO) pour les Wallisiens-Futuniens... Mais peut-on généraliser à l'ensemble d'une communauté ? Le propre d'une grande partie des «victimes de l'histoire " n'est-il pas de partager cette commune condition d'être situées du côté des dominés? Quand bien même, en admettant la position singulière de cette communauté (en lien avec les nationalismes kanak et algériens), jusqu’à quel point peut-on parler de médiation communautaire? En fait, ce ne sont pas les communautés qui se parlent ici, ce sont avant tout des hommes politiques ou des intellectuels qui dialoguent, comme Jean-Pierre Aïfa ou Christophe Sand ici. Ces réflexions ne sont pas tant adressées à l'auteur qu'à une intelligentsia locale - à laquelle Umberto Cugola se reconnaît appartenir - qui aurait la charge de conceptualiser la praxis et le rapport que les gens du peuple calédonien entretiennent avec leur(s) propre(s) histoire(s). Car ceux qui savent écrire et penser le sens de ces vides, de ces 
nostalgies, de ces tragédies dont nous héritons, alimentent l'imaginaire social d'un identique là où l'aliénation coloniale n'a fait qu'entretenir du différent.

Rachid Oulahal, Zohra Guerraoui et Patrick Denoux, spécialistes de psychologie interculturelle, traitent de la question de l'appartenance culturelle plurielle et des processus identitaires qui, s'ils se posent pour l'ensemble des habitants du territoire, ne sont vus ici que pour le cas particulier des descendants d'Algériens en Nouvelle-Calédonie, à partir des résultats d'une recherche qualitative réalisée auprès de neuf d'entre eux. L'histoire, la psychologie interculturelle et l'anthropologie offrent le cadre conceptuel au sein duquel les auteurs structurent leur propos. Comme nous l'avons déjà mentionné, le contexte contemporain du pays réactive ces phénomènes identitaires audelà de la seule communauté kanak. Pour ces descendants d'Algériens, une identité interculturelle se construit autour d'une identité racine et d'une identité néo-calédonienne en devenir.

Même si la méthode présentée (quinze jours d'entretiens menés auprès de seulement neuf personnes) pourra appeler certaines critiques, le résultat de ceux-ci, déroulé judicieusement selon un axe générationnel, donne un fil de sens intéressant dans l'évolution du processus identitaire. Au-delà de ce résultat, le contexte politique de l'époque de cette recherche, de même que la levée du silence quant aux origines et l'identification de liens familiaux en Algérie, peuvent-ils produire une mémoire collective et une émergence diasporique au sein de cette communauté ? Les auteurs en effet se demandent à juste titre si, au regard des enjeux contemporains de l'histoire calédonienne (une décolonisation en cours et un référendum qui a exacerbé les processus identitaires au sein de la mosaïque ethnique existante), nous ne sommes pas en train d'assister à l'émergence d'une diaspora algérienne en Kanaky Nouvelle-Calédonie. Avec une désinhibition sur le "non-dit " d'antan (Barbançon, 1982) des jeunes générations portées par les travaux reconnus d'historiens (locaux ou non), la communauté arabo-calédonienne réorganise son rapport à l'histoire et à ses origines pour construire sa légitimité à occuper une place fondatrice dans le projet calédonien. L'hypothèse de travail des auteurs invite à pousser l'analyse, que l'on retrouve également dans le texte de Christophe Sand, mais sous une autre forme. Ici, les auteurs parlent de diaspora comme objet fondateur d'une communauté en quête d'identité. La diaspora arabo-calédonienne émerge d'expériences et de souffrances. Elle aspire à une mobilité circulaire pour renouer avec un " tertre " des origines depuis lequel un Calédonien (descendant d'Arabe en l'occurrence) se déplace dans les représentations qu'il a de lui-même et donc de l'autre (les Kanak en particulier) et forcément de leur relation. C'est ainsi, nous rappellent les trois auteurs de ce texte tout comme Christophe Sand, qu'un descendant d'Arabe en Kanaky NouvelleCalédonie peut découvrir ce qui le rapproche du peuple kanak.

Avec Claude Grin, est abordé le destin d'une autre communauté, celle des descendants de Japonais. Les émigrants japonais, qui se sont établis en Nouvelle-Calédonie au tournant du XIx et du $\mathrm{Xx}^{\mathrm{e}}$ siècles, ont noué des relations matrimoniales avec des Kanak et avec des descendants d'Européens. Leurs généalogies se trouvent ainsi intriquées avec celles de nombreuses familles non japonaises et ce, sur plusieurs générations, jusqu'au point d'avoir perdu la mémoire de l'histoire de leurs ancêtres japonais arrivés sur le territoire calédonien. À partir d'un travail auprès des familles, des renseignements riches et inédits viennent compléter la sécheresse des archives institutionnelles ou d'entreprises. En s'intéressant plus particulièrement à des membres d'une même famille japonaise originaire d'Okinawa, l'auteure retrace, en reprenant divers témoignages, leur histoire influencée par le poids du pouvoir colonial, et reconstitue un passé resté trop longtemps méconnu, alors qu'il est pourtant représentatif de bien des situations sociales calédoniennes contemporaines. Cet article apporte des réponses à des questions que l'on peut se poser sur le devenir des engagés après leur départ, soit de la Société Le Nickel (SLN), soit du pays. Au-delà de la reconnaissance de cette communauté oubliée, l'auteure donne des éclairages nouveaux et ouvre des pistes de recherche à partir des travaux récents, notamment ceux de l'artiste et universitaire Mutsumi Tsuda. Et comme les archives sont difficilement accessibles car en japonais, ces travaux complètent et enrichissent la mine d'informations que constitue le livre de Kobayashi Tadao (1992).

Pour mesurer la question du rééquilibrage qui découle des accords de Matignon, Jean-Louis Rallu procède à l'élaboration et à l'analyse comparative par communauté ethnique et par provinces, d'un " corpus " statistique qui lui permet de balayer de façon assez exhaustive des secteurs entiers de la vie économique et sociale en Nouvelle-Calédonie : éducation, emploi, pauvreté, santé, habitat, etc. La démonstration est technique mais le débat est clairement posé. L'auteur note qu’à partir des années 2000, le gouvernement de Nouvelle-Calédonie s'est détourné de l'objectif affiché du rééquilibrage avec, dès 2003, le retrait de la question sur l'appartenance communautaire (rétablie en 2009) qui permettait de mesurer les écarts statistiques entre les différentes communautés. L'éducation est la base du rééquilibrage socioéconomique. Mais le passage de l'enseignement primaire, puis secondaire, sous compétence locale, s'est accompagné de stratégies pour réduire l'accès des Kanak aux niveaux bac, et surtout supérieur : moins de $5 \%$ de diplômés de l'université en 2014. Avec le retard de la législation sur l'emploi local (2010), 
l'emploi des Kanak n'a connu qu'une croissance lente, leurs taux d'inactifs et de chômeurs restent plusieurs fois supérieurs à ceux des autres communautés et leur accès aux professions élevées est encore très faible : près de $80 \%$ sont agriculteurs, ouvriers ou employés. Le processus de décolonisation s'est ainsi trouvé enrayé, laissant les Kanak avec les autres Océaniens au bas de la société calédonienne. Après plus de quarante années de lutte et de promesses, accepteront-ils cette intégration par le bas, au mieux en seconde zone pour une petite minorité d'entre eux ?

L'argumentaire posé par l'auteur fait un constat édifiant, trente ans après la signature des accords de Matignon. Le rattrapage du déséquilibre qui, historiquement, affecte les Kanak, ne s'est pas réalisé. Les constats de la statistique sont implacables: l'auteur observe un lien entre le faible niveau de diplôme et un manque d'amélioration du système scolaire. Le constat d'une telle corrélation rejoint une question abordée dans le texte de Stéphane Minvielle : une école pour quel projet de société ? L'introduction de particularismes locaux, de langues locales... constituent une orientation intéressante mais gomme-t-elle pour autant les fondements méritocratiques de l'école républicaine fatalement disqualifiante pour ceux qui s'écartent de la norme de l'élève "idéal " ?

J.-L. Rallu rappelle le fort taux d'illettrisme, surtout aux îles Loyauté et sur la côte Est, accompagné d'un taux de pauvreté assez élevé. On constate un dénuement extrême et sévère en zone urbaine, principalement dans les squats de Nouméa et de sa périphérie, qui peut paraître digne du " tiers monde ", pauvreté qui est traitée par des politiques publiques (sociales, de la ville, d'aménagement...) qui ne cherchent parfois plus à la résorber nécessairement mais peut être à la rendre acceptable, selon les standards des pays dits développés. Le second type de pauvreté, qualifiée "d'opportunité " par l'auteur, est celle que l'on peut observer en zone rurale et dans certaines tribus, combinant un accès à des ressources vivrières et à des solidarités communautaires et familiales, soit tous ces systèmes d'entraide qui permettent de l'amortir largement. Sans eux, cette précarité plus ou moins chronique aurait toutes les chances de basculer vers une situation de pauvreté sévère et d'exclusion classique, ce qui est observable aux abords de Nouméa. Les opportunités d'emploi, quant à elles, sont très liées à une migration hors tribu. Les Kanak doivent sortir de la tribu pour pouvoir travailler, tout en campant au bas de l'échelle sociale :

"les Kanak sont restés au bas de la société de Nouvelle Calédonie, très en-dessous des "autres natifs" et sans grand changement par rapport aux autres Océaniens. Et les choses ne vont pas changer rapidement, vu le travail qui reste à faire pour parvenir à une réduction significative des déséquilibres et la lenteur des progrès observés jusqu’à présent. » (p. 408)
Ces constats invitent à pousser l'analyse en repartant des deux formes de pauvreté identifiées par l'auteur : la première est celle de la ville, une pauvreté classique de notre modernité se manifestant par des critères comme la malnutrition, le mal-logement, un accès précaire aux soins, etc. En terre coutumière, le système communautaire fonctionne comme un amortisseur social dont l'équilibre est cependant menacé par la pression exercée à la fois sur l'organisation sociale des tribus (selon, pour le dire rapidement, un principe assimilationniste : école, salariat et rapport à la ville) et sur l'environnement (en tant qu'il produit des ressources vivrières).

Selon l'auteur, dans la population kanak, nombreux sont les laissés-pour-compte de la croissance qui risquent d'orienter leur vote vers l'indépendance en signe de mécontentement. Rallu se demande aussi si ces laissés-pour-compte ne seront pas tentés par un boycott passif du vote du 4 novembre : un refus de participation de la part de ceux pour qui le vote n'aura absolument aucun impact sur leur condition. Pour eux, voter pourrait revenir à valider un système structurellement excluant à leur égard. Les résultats du référendum ont montré qu'il n'en était rien.

En lien avec le référendum du 4 novembre 2018, cet article porte l'attention sur les interactions entre le social et le politique, à partir des recensements de population et des statistiques qu'ils contiennent. L'histoire durable des faibles taux de diplômés chez les Kanak tout comme les relations entre illettrisme et pauvreté sont des données incontournables, tant pour comprendre la situation actuelle que pour mener des politiques pour les combattre. Ici aussi l'auteur rappelle que la persistance du déséquilibre entre ethnies repose sur le fait qu'une réelle décolonisation du pays n'a jamais été vraiment pensée ou imaginée. En cela, Jean-Louis Rallu rejoint d'autres commentateurs de la situation politique calédonienne réelle, que ce soit Isabelle Leblic, Pierre-Yves Le Meur, Denis Monnerie ou Marie Toussaint, parmi d'autres.

L'article de Rallu doit être mis en regard de celui de Leblic déjà présenté. Il reprend les données statistiques des derniers recensements pour revenir sur la réalité du rééquilibrage et de la décolonisation annoncée dans le cadre de l'accord de Nouméa en mobilisant la statistique, la démographie et les données socio-économiques. En repartant $\mathrm{du}$ fait que les accords de Matignon (1988) ont posé les bases d'une réduction des écarts socioéconomiques entre communautés hérités de la colonisation et que l'accord de Nouméa (1998) a prôné le " vivre ensemble » au sein d'une situation plus équitable, l'auteur examine dans les données chiffrées ce qu'il en est réellement.

Dans le domaine de la statistique ethnique, il existe des controverses intéressantes avec des auteurs comme Patrick Simon, socio-démographe, ou René Otayeck, chercheur en sciences 
politiques. Ce dernier (2000, 2001), par exemple, pose un débat qui montre les bégaiements de l'État toujours mal à l'aise avec la question ethnique, ce qui fut le cas en Nouvelle-Calédonie, notamment quand Jacques Chirac, président de la République, suite à un voyage à Koné, interpellé par un anti-indépendantiste sur la légalité des comptages ethniques en France, avait trouvé cela " proprement scandaleux ", le résultat, en contradiction avec la $\mathrm{loi}^{7}$, fut la suppression des statistiques ethniques dans les recensements de population en 2004. Ces statistiques furent rétablies en 2009 par la question "à quelle(s) communauté(s) estimez-vous appartenir ? " avec neuf possibilités «européenne, indonésienne, mélanésienne, vanuatuaise, tahitienne, vietnamienne, wallisienne-Futunienne, autre asiatique et autre sans précisions ".

Une donnée incontournable pour l'avenir du pays est la question de la monnaie sur laquelle Laîsa Ro'i se penche de façon très pédagogique. En présentant les notions de base relatives à la monnaie et à sa nature, l'auteure se rend accessible à des non-spécialistes d'économie. Elle explique ainsi clairement la politique monétaire contemporaine, les relations entre les dynamiques macro et micro économiques en jeu dans un Etat du Pacifique insulaire, les enchevêtrements entre un ancien ou actuel "partenaire" (si l'on peut dire), colonial et métropolitain et ce futur État, la confrontation dans les paradoxes ou les dilemmes des décisions monétaires par un gouvernement autonome qui pourrait voir (ou être forcé de voir) l'instrument monétaire de l'ancienne puissance coloniale rejeté pour être immédiatement forcé d'adopter l'instrument monétaire d'une autre super-puissance (par exemple des USA, de l'Australie ou de la Nouvelle-Zélande), tout cela est ici exposé magistralement. Tout le paradoxe de la monnaie est que, si elle relève certes d'une question économique, elle est avant tout un enjeu politique au cœur de l'avenir institutionnel de la Kanaky Nouvelle-Calédonie souveraine. Car, en la matière, des choix importants devront être faits par les responsables du futur nouveau pays indépendant : rester dans le giron français et européen ou choisir une politique océanienne et internationale. Pour certains, l'orientation minière de l'économie calédonienne et ses conséquences sur le commerce pourraient faire pencher la balance en faveur de la deuxième option. En général, les pays indépendants choisissent soit la monnaie de leur allié (politique ou économique), soit une monnaie propre, comme l'ont fait la Papouasie NouvelleGuinée, Fidji, ou Vanuatu, qui sont des pays qui, structurellement, restent néanmoins dépendants de capitaux extérieurs. L'analyse de la balance des paiements de la Nouvelle-Calédonie montre l'importance du dollar pour les exportations dont la grande majorité se fait vers l'Asie qui ont des monnaies en régime de change fixe ou en régime intermédiaire vis-à-vis du dollar américain. Pour la Nouvelle-Calédonie actuelle, les subventions de l'État français demeurent importantes. Un des arguments des anti-indépendantistes réside dans le fait que la Kanaky Nouvelle-Calédonie souveraine ne pourrait pas subvenir à ses besoins sans ces subventions. Pour les indépendantistes, il n'en est rien puisqu'une grande partie des subventions reçues repartent en métropole (voir Leblic dans ce volume). En discutant des régimes de change possibles auxquels la Kanaky Nouvelle-Calédonie, si elle parvient à la souveraineté/indépendance, pourrait adhérer, cet article apporte des éléments importants dans la réflexion sur l'avenir institutionnel du pays.

La mine étant au cœur des politiques économiques actuelles du territoire et un point central du projet d'indépendance du pays, l'article de Frank Enjuanes sur la fiscalité minière constitue une porte d'entrée importante dans la compréhension du dossier calédonien. Cette étude retourne aux racines de la fiscalité minière calédonienne et s'organise autour des questionnements contemporains qui lui sont associés. L'étude historique des textes de loi et des données budgétaires, sources riches, claires et accessibles, permet de mettre en lumière les lignes de force de cette histoire. Après quelques tâtonnements, les redevances sur le patrimoine minier et, surtout, la taxation proportionnée à l'exportation se révèlent être des outils majeurs de la fiscalité calédonienne. Cependant, à partir des années 1970, l'imposition sur les bénéfices des sociétés minières devient le principal levier fiscal. La mise en relation des évolutions de la fiscalité minière avec les autres évolutions de la politique minière et aussi les tournants de l'histoire calédonienne permet une compréhension de bien des paramètres de l'évolution politique et économique du territoire et les sources mobilisées permettent de situer les inflexions (ou les continuités) en matière de fiscalité.

L'article de Benoît Carteron, qui emprunte à l'ethnologie et à la sociologie (voire à la psychosociologie), aborde les sociabilités ordinaires dans l'espace périurbain pluriculturel, en lien avec l'appartenance au pays. L'auteur dessine une fresque qui vise à dépeindre des processus identitaires ancrés au territoire des quartiers d'une commune

7. "La loi "informatique et liberté" de 1978, qui interdit les références à l'appartenance ethnique en France métropolitaine, les autorise explicitement pour la Nouvelle-Calédonie dans l'alinéa 3 de son article 31 . Argument retenu : "La Cnil (Commission nationale informatique et liberté) considère que le recueil de l'appartenance ethnique des personnes, compte tenu du caractère socio-démographique propre à la Nouvelle-Calédonie, répond à un motif d'intérêt public." Seule condition : que le Conseil d'État l'autorise par décret. C'est à ce décret que le gouvernement a donné hier un avis favorable. » (https://histoirecoloniale.net/recensement-ethnique-en-Nouvelle.html). 


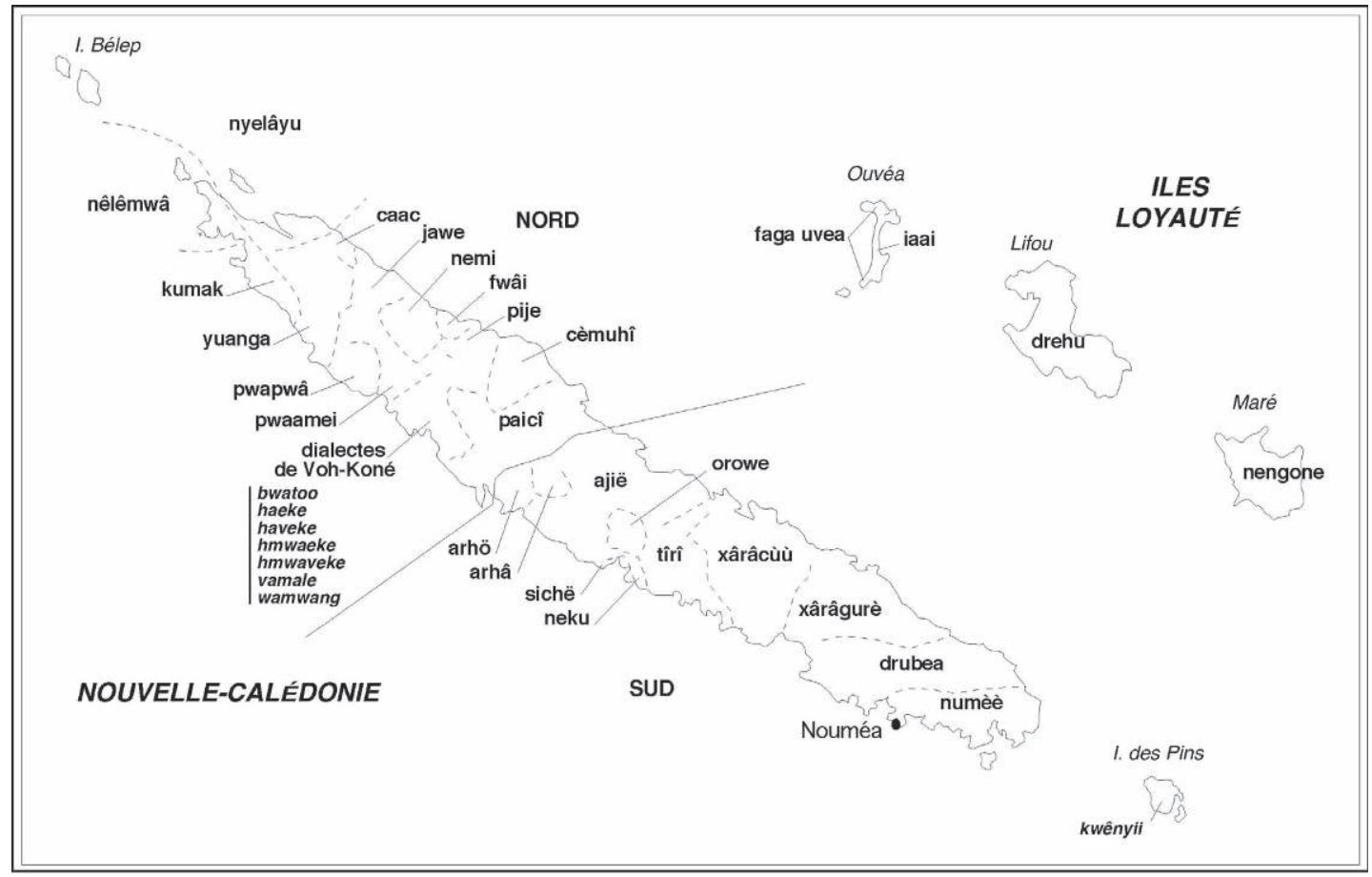

Carte 3. - Carte linguistique de Nouvelle-Calédonie (C CNRS-LACito)

" rurale » du Grand Nouméa. Il s'agit d'une sorte d'historiographie locale fondée à la fois sur des matériaux de terrain riches et documentés et sur des récits de vie. Ces derniers ont été recueillis lors de cinquante-six entretiens et par immersion en trois séjours sur le terrain plus ou moins longs (de un à quatre mois entre 2012 et 2015).

L'auteur retrace, dans le contexte de la commune loyaliste de Dumbéa, où la présence kanak a été très tôt effacée, l'extension de l'habitat à partir des années 1970 au sein du quartier de KatiramonaNondoué (partie nord de cette commune du Grand Nouméa). La part de la population kanak de Dumbéa est passée de 10,73\% en 1983 à $26,67 \%$ en 2014 . Elle est donc en pleine expansion, la plus forte connue pour l'ensemble des communes du Grand Nouméa. Ce quartier de la périphérie de Nouméa, où la majorité des habitants est de condition modeste, connaît une sociabilité familiale et de voisinage singulière. Leurs habitudes communes et la représentation qu'ils ont d'eux-mêmes présentent à l'observateur une société locale unifiée par des traits communs, même si l'investissement dans la vie de quartier contrecarre difficilement le cadre de groupes familiaux et culturels localisés. En se distanciant des clivages politiques du pays, bien qu'avec des choix de société opposés (pour ou contre l'indépendance ou selon qu'on est kanak ou pas), le sentiment d'appartenance reflète cette proximité des groupes par la complexité des identités et la souplesse de leur affirmation.

Ce texte révèle la réalité de la construction en cours du lien social dans un vivre ensemble. Même si les trajectoires singulières ne sont pas indemnes de contradictions, les face-à-face et l'éprouvé de l'expérience humaine au quotidien, avec ses rituels de la rencontre (se dire bonjour, tout simplement), les rituels du cycle de la vie (naissance, mariage, deuil, etc.) obligent chacun à se reconnaître. Tous éprouvent en effet un territoire - les quartiers de Dumbéa - qui a vocation à les réunir de par ce qu'il représente pour chacun (la brousse aux portes de la ville par exemple). Chacun vit la présence de l'autre à travers une sociabilité obligée qui pousse à la sublimation des contradictions particulières et sans laquelle "l'être social " n'est pas possible. Ce texte montre finalement que "l'être ensemble ", une "catégorie de pratiques" au sens de Brubaker cité par l'auteur, créatrice d'une identité commune, est déjà là, tandis que le discours rationalisant, objectivant, politique, sur une "communauté de destin ", une identité commune considérée seulement comme catégorie d'analyse abstraite, est clivant, aliénant et ne donne à voir qu'un horizon indépassable quand on le déplie jusqu'à son terme.

À travers le prisme du "retour au pays natal ", Umberto Cugola explore de très nombreux aspects de la civilisation kanak, en lien avec ceux, en tension, de l'éloignement et d'une reprise de contact informée par la recherche et une forte expérience en milieu éducatif. La dimension réflexive personnelle importante - sans jamais devenir gênante - a avant tout vocation à situer le rapport qui lie cet auteur à son objet (le monde kanak) à partir des sciences humaines et sociales, opérant donc par là une sorte d'épistémologie. 
Ce qu'indique bien le premier sous-titre de son texte : "Parler du monde kanak avec les sciences humaines" (p. 457). Géographe de formation, devenu éducateur de métier puis formateur, l'auteur enseigne l'anthropologie dans le champ de la clinique éducative. Son approche a recours aux outils intellectuels tant de la géographie humaine ou " culturelle » que de la psychologie, qu'il combine à ceux de l'anthropologie et des théories sociologiques. À l'occasion de ce retour au pays et grâce au regard éloigné qu'il y porte puisqu'il habite en France depuis de nombreuses années, ainsi qu'à ses formations universitaires, l'auteur passe en revue plusieurs thèmes. Le premier est la notion d'identité kanak qu'il saisit dans une dimension aliénante en mobilisant les travaux de Fanon. Cette identité ne résume pas la civilisation kanak, le lien à la terre, l'espace de la coutume comme "projet de société de la civilisation kanak ». Elle ne dit rien non plus sur l'évolution et la persistance de la coutume kanak dans le milieu urbain du Grand Nouméa, notamment lors des mariages et des deuils, à l'occasion desquels un travail cérémoniel est effectué. L'auteur observe et vit la coutume en acte et tente de nous faire ressentir l'actualité de la résistance du monde kanak dans un pays djubéa largement submergé par le Grand Nouméa. Mettant son analyse d'une résurgence de la coutume en perspective de son expérience vécue en Europe, il y voit un processus "néguentropique» manifestant une résistance face à la mondialisation et son penchant destructeur. «L'espace de la coutume comme processus néguentropique» (p. 466) est l'une des hypothèses fortes de cette contribution. L'hyper mondialisation détruit la diversité des espèces, assèche les ressources, appauvrit les espaces symboliques pour finalement produire une fragilisation exacerbée du lien social. Parce que la coutume constitue un espace de convivialité fondé sur la filiation, l'alliance mais aussi sur le "sacré ", l'auteur y voit une piste pour traiter de la délinquance juvénile. Pour lui, " le "travail" de la coutume propose en effet des dynamiques sociales dans le creuset desquelles des maillages sont à inventer et à tresser pour créer des espaces de transmission et de réaffiliation ». (p. 467)

La démographie kanak en forte augmentation dans le milieu urbain du Grand Nouméa couplée à la densification résidentielle n'empêchent pas la coutume kanak de perdurer. Si les objets cérémoniels échangés dans les mariages varient de façon significative (dans tous les sens du terme) selon les régions, il est important de voir que ces circulations existent toujours, y compris dans le milieu urbain du Grand Nouméa.

Dans son article " "Je ne sais pas ce que c'est, la coutume" : la coutume kanak par-delà tradition et modernité »(pp. 473-486), Dominik Bretteville traite de l'écologie kanak et de la question de la coutume d'un autre point de vue, celui du cher- cheur extérieur à la société qui la pratique. Voici l'ambition affichée au début de sa contribution :

« la coutume, en tant que système de valeurs, maintient sa cohérence en incorporant des éléments allogènes. En cela, la coutume kanak nous enseigne qu'elle est à même de nourrir une réflexion sur les défis environnementaux, en mobilisant en une même configuration logique les dimensions sociales et cosmiques qui animent les échanges cérémoniels. » (p. 475)

L'auteur s'inspire d'un terrain anthropologique datant des années 1990 mené dans la chefferie paimboa au nord du pays pour tenter de clarifier le concept de coutume et en proposer une représentation en tant que "système de valeur ", suivant en cela la théorie de Louis Dumont. L'auteur utilise habilement la polysémie du mot " coutume" pour développer une réflexion sur les pratiques contrastées de la modernité mondialisée et du monde kanak de Nouvelle-Calédonie. La réflexion sur le référendum de 2018 qui se dégage de ce texte n'est pas contrainte par les seules perspectives ou catégories du "politique». Pour ce texte bâti dans un cadre conceptuel référant à l'anthropologie calédonienne, il croise ses données de terrain à une partie de la bibliographie existante, sur le thème de la parole associée à l'objet (voir les travaux de Leblic et Monnerie), sur celui de la tradition et de la modernité, central dans l'ouvrage d'Isabelle Leblic de 1993, sur la nomination dans les sociétés kanak (Leblic et Monnerie), etc.

Selon l'auteur, le référendum calédonien oppose la modernité, communément associée à la culture occidentale, et la coutume, attribuée au monde kanak. Il précise que, héritée de l'époque coloniale, cette représentation des relations entre cultures traduit, d'une manière générale et s'agissant de sociétés autres, une méconnaissance occidentale - déjà pointée en son temps par Isabelle Leblic (1993) - qui n'est pas étrangère à la crise environnementale planétaire. Il montre que la notion de coutume participe d'un ensemble de représentations qui, dans la culture kanak, relèvent d'autres catégories de pensée que les oppositions occidentales tradition/modernité ou nature/culture. Alors que la crise écologique mondiale tend à reconsidérer ces deux distinctions, les cultures kanak - qui n'ont pas attendu le " progrès » ou « l'économie de marché " pour être des sociétés - empruntent à la modernité suivant la cohérence de leur vie sociale pour, avant tout, maintenir la vitalité de leurs organisations. L'auteur avance le concept de "rapport au cosmos » pour tenter d'approcher la coutume qui montre la singularité des personnes qui la pratiquent, qui sont habitées par elle et qui débouche sur un rapport au monde, aux vivants, aux non-vivants et aux non-humains; ce qui reste une dimension très complexe à appréhender. Mais c'est bien le point de départ à partir duquel on peut mettre en évidence que la coutume implique 
un rapport singulier au monde, à soi-même et à l'autre qui est menacé d'extinction par les forces universalisantes du marché.

L'article de Matteo Gallo et Anna Paini, ethnographique et historiographique, propose une " écoute partagée " de deux différents terrains de recherche, l'une dans le nord de la Grande Terre à Wëté, l'autre à Drueulu, sur l'île de Lifou, avec le but de mieux comprendre comment, dans ce moment historique, des hommes et des femmes kanak se réferrent et négocient leur propre passé. Ce travail à quatre mains mobilise ces deux disciplines avec une différence de durée de terrain, puisque A. Paini est arrivée à Lifou bien avant M. Gallo à Wëte. Ce faisant, le travail d'analyse de la première est adossé à une observation sur le long terme bien que parcourue d'interruptions, et propose des interprétations plus en profondeur sur ce qui se joue dans l'articulation de la coutume et des deux religions chrétiennes présentes sur l'île. Grâce à ce comparatisme, la vision binaire opposant les gens des îles Loyauté et ceux de la Grande Terre, qui a trop longtemps dominé les études anthropologiques calédoniennes et ne permet pas d'expliquer la richesse et la complexité des différentes réalités ethnographiques, se trouve dépassée. Cette approche expérimentale a permis de saisir des différences et des convergences entre les deux contextes locaux pour souligner des tendances aptes à rendre compte de ce moment historique d'un pays en marche vers un «destin commun ". Le texte met ainsi en évidence une sensibilité patrimoniale et une envie accrue des Kanak d'aujourd'hui d'être les protagonistes actifs du redéploiement, de la valorisation et de la réécriture des éléments de leur propre passé.

Ce texte montre aussi comment les Kanak, dans deux contextes différents, mobilisent des éléments de leur passé, de leur patrimoine, pour les inscrire dans une narration contemporaine et, finalement, donner sens au présent. Il en est ainsi de la démarche de l'association Pi Wian Mââ Paarii dans la tribu de Wété, suite à la fermeture de l'école publique de proximité, qui a eu pour effet d'éloigner les jeunes scolarisés du milieu local (et « local "par opposition au "global ", " en tant que catégories subjectives », voir Dimitrova, 2005), privant ainsi la tribu de dynamiques de transmission à même de composer et d'articuler le particulier et le " global ». L'association a réutilisé ce lieu et l'a transformé en un lieu de transmission de l'histoire et du patrimoine de la tribu (accueil de scolaires, de visiteurs, etc.) (p. 492). De même, la "réforme du mariage » à Lifou (p. 494) est un phénomène intéressant qui se retrouve sous d'autres formes ailleurs dans la Grande Terre. Les îles Loyauté ayant été beaucoup plus préservées que la Grande Terre du contexte colonial, cette modération des discours à Lifou paraît justifiée face au discours plus virulent, par exemple, dans le sud où le rejet des "objets non authentiques" se pose sur un ton beaucoup plus vindicatif et "militant $"^{8}$. On retrouve en écho l'idée que les processus de remobilisation patrimoniaux sont toujours liés à l'héritage colonial et à ses meurtrissures. La spoliation foncière violemment vécue sur la Grande Terre entraîne la mobilisation du patrimoine dans un discours et des pratiques beaucoup plus offensifs. Même si, au fond, comme à Lifou, la réflexion sur la consommation et le modernisme est bien ce qui se joue en arrière-plan.

Il est vrai que cette reformulation implique aussi une ré-articulation entre chrétienté et coutume. Mais c'est surtout le fait politique qu'il faut prendre en considération, voire même le fait colonial, car c'est en raison d'une conscience politique et une vraie conscience des enjeux modernes que ce qui paraissait immuable le devient vraiment. Car au fond, ce sont deux visions du monde sur une façon d'être au monde ou en tout cas de faire société, de construire et nourrir le lien social... qui s'opposent'. Le propos de Paini et Gallo entre dans un débat sur un enjeu majeur pour les Kanak du Xxi ${ }^{e}$ siècle : dans quelle mesure peuton dire que le rapport qu'ils entretiennent avec la mémoire, l'histoire, le patrimoine et, plus largement, leurs traditions, dessine les limites d'une lutte qui, au-delà d'être anticolonialiste, est aussi civilisationnelle?

Une question qui se trouve toujours depuis plus de trente années de rééquilibrage au cour des débats sur l'inégalité des chances dans le jeu social et politique de la pluralité ethnique est l'école. Stéphane Minvielle, quant à lui, revient sur le projet éducatif calédonien voté au début de l'année 2016 par le congrès de la Nouvelle-Calédonie, suite à une dizaine d'années de négociations, puis sur sa mise en œuvre concrète sous la forme d'une Charte d'application (plan d'action triennal) votée à la fin de l'année 2016 et entrée en vigueur lors de la rentrée scolaire de février 2017. L'arrière-plan théorique de ce texte est issu des sciences de l'éducation, avec des objets centrés non seulement autour de l'école et des processus d'éducation, mais aussi plus largement de la transmission, de la citoyenneté, de la place de la diversité linguistique et culturelle dans un contexte de décolonisation. L'intérêt de cette proposition tient au travail de reconstruction d'un fil historique, avec ses enjeux, qui a mené à l'adoption de ce

8. Ainsi, Umberto Cugola présente un phénomène analogue pour les tribus de Saint-Louis et de La Conception, auxquelles on peut aussi ajouter Paita. Les objets " authentiques ", c'est-à-dire ceux auxquels une dimension commerciale est moins associée, sont préférés aujourd'hui dans les coutumes (les nattes et les tapas fabriqués localement remplacent les étoffes " asiatiques ").

9. Voir à ce propos aussi Cugola dans ce volume. 
Projet éducatif de la Nouvelle-Calédonie (PENC) en prenant pour point de départ les périodes de transferts de compétences de 2000 et de 2012. La genèse du PENC restituée très précisément ici l'est sur un style descriptif et les statistiques et dates mobilisées sont un matériau qui appelle indubitablement à la réflexion et à l'analyse. En saisissant dès la genèse du PENC la négociation de la place des langues et de la culture kanak avec ses déterminants sociaux, culturels, historiques et politiques, l'auteur cherche à comprendre un processus à travers lequel il tente d'éclairer une question centrale de sa proposition : le PENC et les textes qui lui succèdent peuvent-ils être considérés comme «l'acte de naissance d'une école décolonisée/émancipée, à l'identité propre et capable de répondre aux besoins d'une société insulaire du Pacifique?» (p. 501). L'auteur positionne l'enjeu du débat autour des notions suivantes: école inadaptée ou non, école en rupture avec l'école coloniale ou non, école plus ou moins en continuité avec le modèle national, etc. À partir de là et à juste titre, pour lui, l'école calédonienne, revendiquant une "identité propre", tient du "slogan politique» (p. 511). Tout ne se résume pourtant pas aux "obstacles internes et externes qui freinent indéniablement le processus " qu'il a tentés de mettre en évidence dans ce texte. $\mathrm{La}$ vocation d'un projet d'école émancipée n'est-elle pas avant tout de soutenir un projet de société luimême émancipé (de la puissance colonisatrice par exemple) ? L'expérience des Écoles populaires kanak (EPK) se situait dans ce registre de projets, qui pensent l'école en lien avec une autre vision de la société et de l'épanouissement de la personne en son sein. Si la recherche n’identifie pas les représentations et les pratiques locales alternatives qu'il s'agirait de mettre en évidence en Kanaky Nouvelle-Calédonie pour les associer au débat public sur une école émancipée, penser et poser la question d'une école avec une "identité propre » dans le cadre strict du processus institutionnel en cours ne sera rien d'autre qu'un effet de rhétorique de la part des observateurs et des analystes locaux. Car, au fond, la "vraie» question n'est pas de savoir si le modèle d'école (via le PENC) en gestation répondra à des " besoins hypothétiquement insulaires ", mais s'il relève le défi d'accompagner un projet de société émancipé de son modèle "développementiste ${ }^{10} »$ de tutelle ${ }^{11}$.

Eddy Banaré, enfin, présente, du point de vue de la recherche littéraire, le retour des reliques d'Ataï en septembre 2014 avec un inventaire et une confrontation des discours et récits suscités par la révolte de 1878 et la personnalité du grand chef. L'événement de la restitution est, en effet, travaillé par des processus mémoriels complexes.
Depuis la fin des années 1960, les représentations du grand chef Ataï s'affichent en Nouvelle-Calédonie, d'abord dans les tracts politiques des premiers militants politiques kanak, puis après 1984 , sur des vêtements de toutes sortes, sur les murs de la ville blanche ou les abribus le long des routes territoriales sillonnant la Grande Terre, dans de nombreux logements habités par des Kanak, etc. La figure d'Ataï est donc réapparue en même temps que les revendications indépendantistes kanak se faisaient jour. Tout cela se situe dans le prolongement d'une création littéraire à double affluent - l'un colonial, l'autre kanak - dont les liens et les dynamiques doivent également être décrits à la lumière du projet politique dans lequel s'inscrit la Kanaky Nouvelle-Calédonie de demain. En analysant la présence d'Ataï dans le discours politique et en revenant sur la constitution de deux grands récits, kanak et colonial, l'auteur tente de saisir les transferts et les réappropriations qui ont pu s'opérer entre les écritures coloniales et la création littéraire contemporaine.

Aujourd'hui, cette image, qui montre le signe d'un rapport au passé colonial, est combinée à celle du drapeau de la Kanaky qui a été, pendant la campagne référendaire, un signe fort de ralliement, notamment pour les jeunes générations (voir la campagne «Affichons nos couleurs », dans "En guise de conclusion... " p. 578). Au moment des Événements de 1984, les graffitis représentant Ataï fleurissaient partout au détour des routes du pays kanak, comme le signe fédérateur de la génération de militants de l'époque, rejoint après son assassinat en janvier 1985 par la figure d'Éloi Machoro. Maintenant, c'est le drapeau de la Kanaky qui est mis en scène dans tout le pays kanak, et même au-delà puisque l'on en voit sur Facebook des images virales aux quatre coins du monde (Bruxelles, Pays Bas, UsA, Chine, Thaïlande, Afghanistan, Bali, Rapa Nui, Israël, Jordanie et même dans les manifestations des gilets jaunes..., https://www.facebook.com/pages/category/Art/Affichons-Nos-Couleurs-537707940032706/).

\section{Conclusion}

Ce dossier, qui a été voulu très en phase avec l'actualité, a donc regroupé plusieurs chercheurs de disciplines différentes apportant tous un regard sur certaines questions au cœur du processus institutionnel en cours en Kanaky Nouvelle-Calédonie. Bien sûr, il reste beaucoup de domaines à approfondir, voire même à explorer et nous comptons bien poursuivre ce débat dans les volumes à venir d'ici le prochain référendum de 2022.

10. Voir en la matière les travaux de Serge Latouche, François Partant...et, sur le Nouvelle-Calédonie, d'Isabelle Leblic.

11. Dans le mini dossier de fin 2019, les textes de Razafi et de Wadrawane apporteront un autre éclairage sur ces questions. 


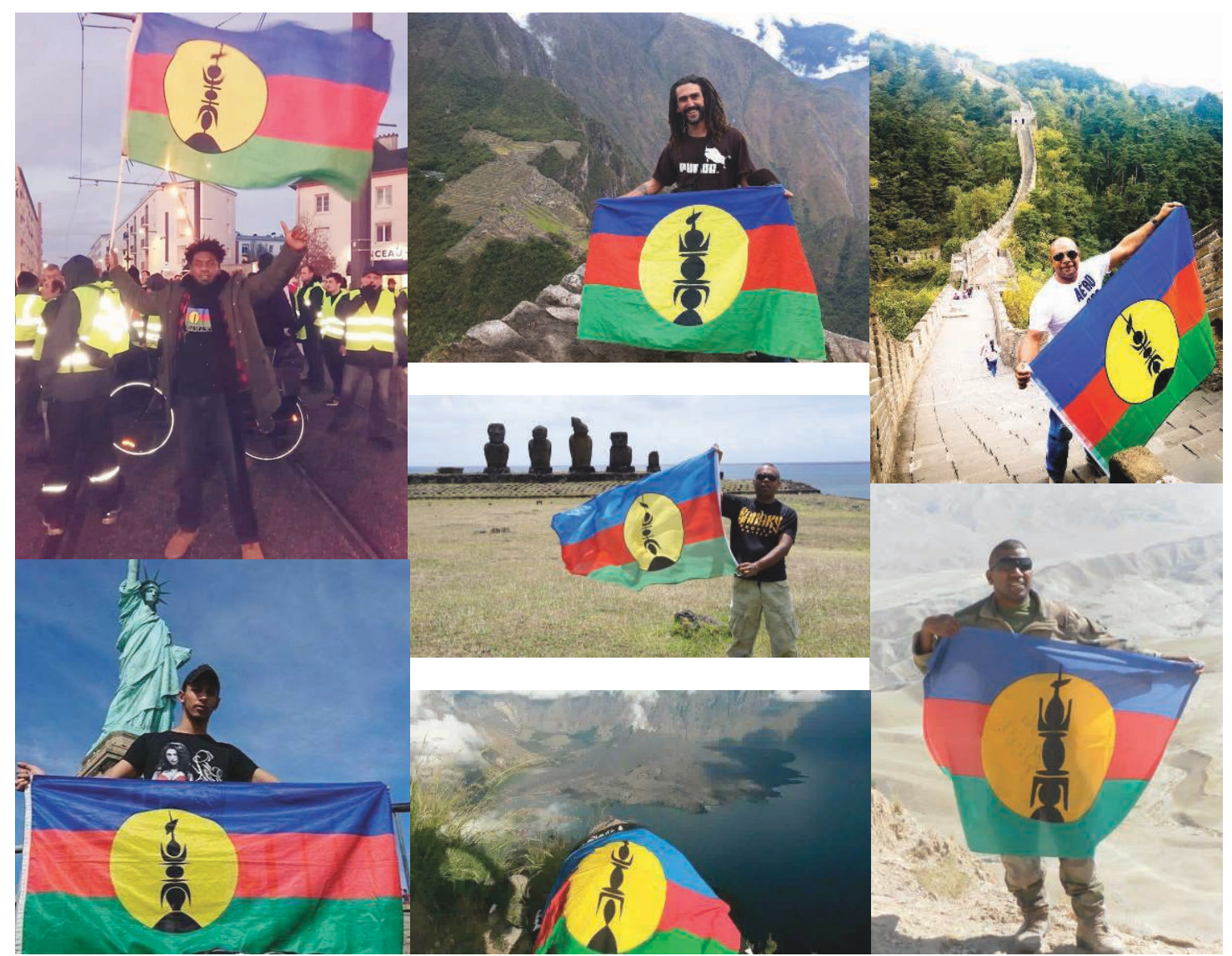

Рнотол 2-8. - Facebook « Affichons nos couleurs » : « Le drapeau knky a décidé de faire le tour du monde! ». Manifestation des gilets jaunes du 16/12/2018; à New York : statue de la Liberté ; au Pérou : Machu Pichu ; à Rapanui ; à Bali : à 3726 mètres au volcan Rindjani Lombok ; en Chine : la Grande Muraille ; en Afghanistan...

\section{Remerciements}

Les auteurs remercient Pascale Bonnemère et Denis Monnerie pour leur lecture et pour les commentaires et propositions d'améliorations apportées. Ils restent néanmoins responsables de ce qui est écrit ici.

\section{BIBLIOGRAPHIE}

Anonyme, 2018. Désindexation, quel impact pour notre pays ?, La voix de Kanaky 9, p. 7

Argounès Fabrice, Sarah Mohamed-Gaillard et Luc VACHER, avec Cécile Marin (cartographe), 2011. Atlas de l'Océanie. Continent d'îles, laboratoire du futur, Paris, Autrement.

Barbançon Louis-José, 1992. Le Pays du NonDit, Regards sur la Nouvelle-Calédonie, Nouméa, édition à compte d'auteur.

-, 2008. Il y a 25 ans : le gouvernement Tjibaou (18 juin 1982-18 novembre 1984), Nouméa, Centre culturel Tjibaou, Cahiers des conférences de l'ADCK 11.
Castoriadis Cornelius, 1996. La Montée de l'insignifiance, les Carrefours du labyrinthe IV, Paris, Seuil.

Collectif, 1994. Etre Caldoche aujourd'hui, Nouméa, Île de Lumière, 256 p.

—, 1996. Notre pays demain, éditions du Cagou, Nouméa.

Cugola Umberto, 2017. Malaise dans la colonisation. Pour une clinique de la délinquance juvénile en pays kanak, Journal de la Société des Océanistes 144-145, pp. 221-238 (https:// journals.openedition.org/jso/7724).

Dimitrova Anna, 2005. Le « jeu» entre le local et le global : dualité et dialectique de la globalisation, Socio-anthropologie 16 (http://journals. openedition.org/socio-anthropologie/440).

Douyère Éric, 1997. Nouvelle-Calédonie: Quel avenir pour l'indépendance? ou Du rééquilibrage des idées (petit lexique pertinent), Nouméa, Île de Lumière.

—, 2000. L'avenir de la Nouvelle-Calédonie en question(s) ou Du rééquilibrage des idées (II), Nouméa, île de Lumière. 
JSO, 1953. Journal de la Société des Océanistes 9, le centième anniversaire de l'annexion du pays par la France (https://journals.openedition. org/jso/persee-246481).

-, 1995. Journal de la Société des Océanistes 100101: Double anniversaire (http://www.persee. $\mathrm{fr} /$ issue/jso_0300-953x_1995_num_100_1? section $\bar{d}=$ jso_0 $300-9 \overline{5} 3 x_{-} 1995$ _ num_100_1_1944).

KobaYashi Tadao, 1992. Les Japonais en NouvelleCalédonie. Histoire des émigrés sous contrat, traduction Raulet-Akaza Keiko, Nouméa, Société d'études historiques de la Nouvelle-Calédonie, publications 48.

Kurtovich Nicolas. Notre pays demain, Nouméa, éditions du Cagou.

LeBLIC Isabelle, 1993. Les Kanak face au développement. La voie étroite, Grenoble, Presses universitaires de Grenoble.

-, 2002. Ignames, interdits et ancêtres en Nouvelle-Calédonie, Journal de la Société des Océanistes 114-115: En hommage à Jacques Barrau (C. Coiffier éd.), pp. 115-127 (https://journals.openedition.org/jso/1439).

-, 2003a. Présentation 150 ans de colonisation en Nouvelle-Calédonie, Journal de la Société des Océanistes 117 (I. Leblic éd.), pp. 135-145 (https://journals.openedition.org/jso/1242).

—, 2005. Pays, "surnature " et sites "sacrés" paicî à Ponérihouen (Nouvelle-Calédonie), Journal de la Société des Océanistes 120-121: Ethnoécolonie en Océanie, pp. 95-111 (https:// journals.openedition.org/jso/410).

—, 2018a. En guise de conclusion. Une Kanaky Nouvelle-Calédonie souveraine en marche?, JSO 147 : 2018. La Kanaky Nouvelle-Calédonie a rendez-vous avec l'Histoire (I. Leblic et U. Cugola éds), pp. 577-588.
-, 2018b. CR de L.-J. Barbançon, Il y a 25 ans : le gouvernement Tjibaou (18 juin 1982-18 novembre 1984), JSO 147: 2018. La Kanaky Nouvelle-Calédonie a rendez-vous avec l'Histoire (I. Leblic et U. Cugola éds), pp. 592-595.

-, 2018c. CR de J.-L. Mathieu, La Nouvelle-Calédonie sera-t-elle indépendante? Analyse d'une politique publique, JSO 147 : 2018. La Kanaky Nouvelle-Calédonie a rendez-vous avec l'Histoire (I. Leblic et U. Cugola éds), pp. 596-599.

-, 2018d. CR de H. Mokaddem, L'accord de Nouméa pour tous, JSO 147 : 2018. La Kanaky Nouvelle-Calédonie a rendez-vous avec l'Histoire (I. Leblic et U. Cugola éds), pp. 595-596.

Lebuic Isabelle (éd.), 2003b. Journal de la Société des Océanistes 117 : Nouvelle-Calédonie, 150 ans après la prise de possession (https://journals. openedition.org/jso/6837).

-, 2013. Journal de la Société des Océanistes 136137 : La part d' immatériel" dans la culture "matérielle" (https://journals.openedition. org/jso/6837).

Mathieu Jean-Luc, 2018. La Nouvelle-Calédonie sera-t-elle indépendante? Analyse d'une politique publique, Paris, L'Harmattan.

Mokaddem Hamid, 2011. L'accord de Nouméa pour tous, Nouméa, Institut de formation des maittres de la Nouvelle-Calédonie, Publications des conférences de l'ifmNC.

Monnerie Denis, 2005. La parole de notre Maison. Discours et cérémonies kanak aujourd'hui, Paris, CNRs Éditions / Éditions de la maison des sciences de l'homme, coll. Chemins de l'ethnologie.

Paturet Jean-Bernard, 2001. L'intervention sociale : au nouage de l'éthique et du politique, in Rémy Puyuelo, Penser les pratiques sociales, Toulouse, éditions Érès, coll. Éducation - Formation, pp. 231-237 (DOI 10.3917/eres. puyue.2001.01.0231). 